\title{
Accurate BER Analysis of Square OSTBCs With Imperfect Channel Estimation in Arbitrarily Correlated Rayleigh Fading
}

\author{
Lennert Jacobs and Marc Moeneclaey \\ Ghent University, TELIN Department, DIGCOM Group \\ Sint-Pietersnieuwstraat 41, B-9000 Gent, Belgium \\ \{Lennert.Jacobs,Marc.Moeneclaey\}@telin.ugent.be
}

\begin{abstract}
In this contribution, we present a novel closed-form approximation of the bit error rate (BER) for square orthogonal space-time block codes (OSTBCs) under arbitrarily correlated Rayleigh fading with imperfect channel estimation. Although derived for a mismatched maximum-likelihood receiver that obtains the channel state information through pilot-based linear minimum mean-square error (LMMSE) channel estimation, the presented expression is shown to yield very accurate BER results for both LMMSE and least-squares channel estimation, over a wide range of signal-to-noise ratios. The information symbols are assumed to belong to a pulse amplitude modulation or square quadrature amplitude modulation constellation.
\end{abstract}

\section{INTRODUCTION}

The exponential growth in wireless communication services has triggered an ever increasing demand for higher data rates and better error performance. By using multiple antennas at the transmitter and receiver side, multiple-input multipleoutput (MIMO) wireless systems can significantly increase the capacity of a radio link [1], [2]. In order to improve the error performance of MIMO systems, they are often combined with space-time coding, which takes advantage of both spatial and temporal diversity. Particularly appealing is the transmit diversity technique of orthogonal space-time block coding [3]-[5], which achieves full spatial diversity and leads to a remarkably simple symbol-by-symbol decoding algorithm, based on linear processing at the receiver.

Owing to the beneficial properties of orthogonal spacetime block codes (OSTBCs), their error performance has been studied extensively in the past. Exact bit error rate (BER) expressions for pulse amplitude modulation (PAM), quadrature amplitude modulation (QAM), and phase-shift keying (PSK) constellations were presented in [6], under the assumption of correlated Rayleigh fading channels with perfect channel state information (PCSI). In [7], asymptotic error-rate formulas were derived for arbitrarily correlated Rician MIMO channels, in terms of the diversity and coding gains. In practical wireless applications, however, the assumption of PCSI is not valid and the receiver has to estimate the channel response. For square OSTBCs, an exact closed-form BER expression was presented in [8] for PAM and QAM constellations operating over independent and identically distributed (i.i.d.) Rayleigh fading channels with linear minimum mean-square error (LMMSE) channel estimation. In [9], a closed-form expression for the pairwise error probability (PEP) of space-time block codes was derived for arbitrarily correlated Ricean fading channels with imperfect channel estimation (ICE). In this contribution, we extend the result from [8] to arbitrarily correlated Rayleigh fading with imperfect channel estimation. Introducing a high signal-to-noise ratio (SNR) approximation of the channel error covariance matrix, we derive a novel closed-form BER expression for a mismatched maximum-likelihood (ML) receiver that obtains the channel state information through pilot-based LMMSE channel estimation. Moreover, we show that the presented expression yields very accurate BER results for both LMMSE and least-squares (LS) channel estimation, over a wide range of signal-to-noise ratios. The information symbols are assumed to belong to a PAM or square QAM constellation.

We denote by $\operatorname{vec}(\mathbf{X})$ the vector that is obtained by stacking the columns of the matrix $\mathbf{X}$, and by $\mathbf{A} \otimes \mathbf{B}$ the Kronecker product of the matrices $\mathbf{A}$ and $\mathbf{B}$. The norm of a vector $\mathbf{a}$ is denoted by $\|\mathbf{a}\|$.

\section{SignAl MODEL}

Let us consider a wireless MIMO communication system with $L_{\mathrm{t}}$ transmit and $L_{\mathrm{r}}$ receive antennas, employing square OSTBCs from complex orthogonal designs [4], [5]. In this way, each transmitted code matrix is defined by an $L_{\mathrm{t}} \times L_{\mathrm{t}}$ coded symbol matrix $\mathbf{C}$, the entries of which are linear combinations of $N_{\mathrm{s}}$ information symbols $s_{i}=s_{i, \mathrm{R}}+j s_{i, \mathrm{I}}$, $1 \leq i \leq N_{\mathrm{S}}$, with $s_{i, \mathrm{R}}$ and $s_{i, \mathrm{I}}$ denoting the real and imaginary parts of $s_{i}$, respectively, and their complex conjugate $s_{i}^{*}$, such that

$$
\mathbf{C}=\sum_{i=1}^{N_{\mathrm{s}}}\left(\mathbf{C}_{i} s_{i}+\mathbf{C}_{i}^{\prime} s_{i}^{*}\right),
$$

where the $L_{\mathrm{t}} \times L_{\mathrm{t}}$ matrices $\mathbf{C}_{i}$ and $\mathbf{C}_{i}^{\prime}$ comprise the coefficients of $s_{i}$ and $s_{i}^{*}$, respectively. Since scaling does not affect the orthogonality of the code matrix $\mathbf{C}$, we assume that $\mathbf{C}$ is scaled in such way that it satisfies the following orthogonality condition

$$
\mathbf{C}^{H} \mathbf{C}=\mathbf{C} \mathbf{C}^{H}=\lambda\|\mathbf{s}\|^{2} \mathbf{I}_{L_{\mathrm{t}}},
$$

where $\lambda \triangleq L_{\mathrm{t}} / N_{\mathrm{s}}, \mathbf{s}=\left[s_{1}, s_{2}, \ldots, s_{N_{\mathrm{s}}}\right]^{T}$ is the data symbol vector, and $\mathbf{I}_{L_{\mathrm{t}}}$ denotes the $L_{\mathrm{t}} \times L_{\mathrm{t}}$ identity matrix. 
Data transmission is organized in frames consisting of $K_{\mathrm{p}}$ known pilot symbols and $K$ coded data symbols per transmit antenna, with $K$ being a multiple of $L_{\mathrm{t}}$. In this way, $K / L_{\mathrm{t}}$ coded data matrices $\mathbf{C}(k), 1 \leq k \leq K / L_{\mathrm{t}}$, are sent within one frame. To recover the channel state information we use orthogonal pilot sequences, i.e., the $L_{\mathrm{t}} \times K_{\mathrm{p}}$ pilot matrix $\mathbf{C}_{\mathrm{p}}$ has orthogonal rows such that

$$
\mathbf{C}_{\mathrm{p}} \mathbf{C}_{\mathrm{p}}^{H}=K_{\mathrm{p}} \mathbf{I}_{L_{\mathrm{t}}} .
$$

The $L \triangleq L_{\mathrm{t}} L_{\mathrm{r}}$ MIMO channel coefficients are represented by the $L_{\mathrm{r}} \times L_{\mathrm{t}}$ complex-valued random matrix $\mathbf{H}$, which is assumed to remain constant during the length of one frame of $K+K_{\mathrm{p}}$ symbols. Hence, the receiver separately observes the $L_{\mathrm{r}} \times L_{\mathrm{t}}$ matrices

$$
\mathbf{R}(k)=\sqrt{E_{\mathrm{s}}} \mathbf{H} \mathbf{C}(k)+\mathbf{W}(k),
$$

with $1 \leq k \leq K / L_{\mathrm{t}}$, and the $L_{\mathrm{r}} \times K_{\mathrm{p}}$ matrix

$$
\mathbf{R}_{\mathrm{p}}=\sqrt{E_{\mathrm{p}}} \mathbf{H} \mathbf{C}_{\mathrm{p}}+\mathbf{W}_{\mathrm{p}}
$$

where the additive channel noise matrices $\mathbf{W}(k)$ and $\mathbf{W}_{\mathrm{p}}$ affecting the transmission of the data and pilot symbols, respectively, consist of i.i.d. zero-mean (ZM) circularly symmetric complex Gaussian (CSCG) random variables (RVs) with variance $N_{0}$. In the remainder of the paper, we will omit the block index $k$ in (4a) for notational convenience. Using a normalized symbol constellation, i.e., $\mathbb{E}\left[\left\|s_{i}\right\|^{2}\right]=1$, it follows from (2) and (3) that $E_{\mathrm{s}}$ and $E_{\mathrm{p}}$ in (4a) and (4b) denote the average data and pilot symbol energy, respectively. Stacking the elements of the channel matrix $\mathbf{H}$ into the column vector $\mathbf{h} \triangleq \operatorname{vec}(\mathbf{H})$, the elements of $\mathbf{h}$ are arbitarily correlated ZM CSCG RVs with a positive definite covariance matrix $\mathcal{R}_{\mathbf{h h}} \triangleq \mathbb{E}\left[\mathbf{h} \mathbf{h}^{H}\right]$.

Introducing the $L$-dimensional column vectors $\mathbf{r} \triangleq \operatorname{vec}(\mathbf{R})$ and $\mathbf{w} \triangleq \operatorname{vec}(\mathbf{W})$, and $\mathbf{r}_{\mathrm{p}} \triangleq \operatorname{vec}\left(\mathbf{R}_{\mathrm{p}}\right)$ and $\mathbf{w}_{\mathrm{p}} \triangleq \operatorname{vec}\left(\mathbf{W}_{\mathrm{p}}\right)$, respectively, allows us to construct a vector signal model which is equivalent to (4a) and (4b) [9]

$$
\begin{aligned}
\mathbf{r} & =\sqrt{E_{\mathrm{s}}} \mathbf{B} \mathbf{h}+\mathbf{w}, \\
\mathbf{r}_{\mathrm{p}} & =\sqrt{E_{\mathrm{p}}} \mathbf{B}_{\mathrm{p}} \mathbf{h}+\mathbf{w}_{\mathrm{p}},
\end{aligned}
$$

where $\mathbf{B} \triangleq \mathbf{C}^{T} \otimes \mathbf{I}_{L_{\mathrm{r}}}$ and $\mathbf{B}_{\mathrm{p}} \triangleq \mathbf{C}_{\mathrm{p}}^{T} \otimes \mathbf{I}_{L_{\mathrm{r}}}$. From (2) and (3), it follows that $\mathbf{B}^{H} \mathbf{B}=\mathbf{B} \mathbf{B}^{H}=\lambda\|\mathbf{s}\|^{2} \mathbf{I}_{L_{\mathrm{t}}}$ and $\mathbf{B}_{\mathrm{p}}^{H} \mathbf{B}_{\mathrm{p}}=$ $K_{\mathrm{p}} \mathbf{I}_{L}$, respectively.

\section{IMPERFECT CHANNEL ESTIMATION}

By increasing the number of pilot symbols $K_{\mathrm{p}}$ and/or the pilot energy $E_{\mathrm{p}}$, very accurate channel estimation can be obtained. However, the more energy is allocated to the pilot symbols, the less energy remains available for data transmission. With $E_{\mathrm{b}}, \gamma \triangleq E_{\mathrm{p}} / E_{\mathrm{s}}, M$, and $\rho \triangleq N_{\mathrm{s}} / L_{\mathrm{t}}^{2}$ denoting the energy per information bit, the ratio of $E_{\mathrm{p}}$ to $E_{\mathrm{s}}$, the constellation size, and the code rate, respectively, it can be shown that

$$
E_{\mathrm{s}}=\frac{K}{K+\gamma K_{\mathrm{p}}} \rho \log _{2}(M) E_{\mathrm{b}},
$$

which is a decreasing function of $K_{\mathrm{p}}$.

\section{A. LMMSE channel estimation}

Using the vector signal model (5b), the LMMSE channel estimate is given by [9]

$$
\hat{\mathbf{h}}_{\mathrm{MMSE}}=\frac{\sqrt{E_{\mathrm{p}}}}{N_{0}}\left(\mathbf{I}_{L}+\frac{K_{\mathrm{p}} E_{\mathrm{p}}}{N_{0}} \boldsymbol{\mathcal { R }}_{\mathbf{h h}}\right)^{-1} \boldsymbol{\mathcal { R }}_{\mathbf{h h}} \mathbf{B}_{\mathrm{p}}^{H} \mathbf{r}_{\mathrm{p}} .
$$

It is readily verified that the channel vector $\mathbf{h}$ can be written as the sum of the LMMSE channel estimate $\hat{\mathbf{h}}_{\text {MMSE }}$ and a noise term $\varepsilon$ that is statistically independent of $\hat{\mathbf{h}}_{\text {MMSE}}$ :

$$
\mathbf{h}=\hat{\mathbf{h}}_{\mathrm{MMSE}}+\boldsymbol{\varepsilon} \text {. }
$$

Moreover, the following properties can be derived for $\hat{\mathbf{h}}_{\mathrm{MMSE}}$ and $\varepsilon$ :

- The components of $\hat{\mathbf{h}}$ are ZM CSCG RVs, the covariance matrix of which is given by with

$$
\begin{aligned}
\boldsymbol{\mathcal { R }}_{\hat{\mathbf{h}} \hat{\mathbf{h}}} & \triangleq \mathbb{E}\left[\hat{\mathbf{h}}_{\mathrm{MMSE}} \hat{\mathbf{h}}_{\mathrm{MMSE}}^{H}\right] \\
& =\boldsymbol{\mathcal { R }}_{\mathbf{h h}} \boldsymbol{\mathcal { R }}_{\mathbf{h h}}\left(\boldsymbol{\mathcal { R }}_{\mathbf{h h}}+\frac{N_{0}}{K_{\mathrm{p}} E_{\mathrm{p}}} \mathbf{I}_{L}\right)^{-1} .
\end{aligned}
$$

- The components of $\varepsilon$ are ZM CSCG RVs, the covariance matrix of which is given by

$$
\boldsymbol{\mathcal { R }}_{\boldsymbol{\varepsilon} \varepsilon} \triangleq \mathbb{E}\left[\varepsilon \varepsilon^{H}\right]=\boldsymbol{\mathcal { R }}_{\mathbf{h h}}\left(\mathbf{I}_{L}+\frac{K_{\mathrm{p}} E_{\mathrm{p}}}{N_{0}} \boldsymbol{\mathcal { R }}_{\mathbf{h h}}\right)^{-1}
$$

Note that for high SNR, $\mathcal{R}_{\hat{\mathrm{h}} \hat{\mathrm{h}}}$ reduces to $\boldsymbol{\mathcal { R }}_{\mathrm{hh}}$ and the elements of the channel noise vector $\varepsilon$ can be considered as i.i.d. ZM CSCG RVs with variance $N_{0} /\left(K_{\mathrm{p}} E_{\mathrm{p}}\right)$, since $\boldsymbol{\mathcal { R }}_{\varepsilon \varepsilon}$ becomes

$$
\mathcal{R}_{\varepsilon \varepsilon} \approx \frac{N_{0}}{K_{\mathrm{p}} E_{\mathrm{p}}} \mathbf{I}_{L}, \quad \frac{K_{\mathrm{p}} E_{\mathrm{p}}}{N_{0}}>>1 .
$$

\section{B. LS channel estimation}

Contrary to the LMMSE channel estimate, the LS channel estimate does not make use of the a priori channel statistics and is given by [9]

$$
\hat{\mathbf{h}}_{\mathrm{LS}}=\frac{\mathbf{B}_{\mathrm{p}}^{H} \mathbf{r}_{\mathrm{p}}}{K_{\mathrm{p}} \sqrt{E_{\mathrm{p}}}} .
$$

Note that the LMMSE channel estimate (7) reduces to the LS channel estimate (12) in case of high SNR. More specifically, LS and LMMSE channel estimation will yield similar performance as long as the diagonal elements of $\boldsymbol{\mathcal { R }}_{\mathbf{h h}}$ multiplied by $K_{\mathrm{p}} E_{\mathrm{p}} / N_{0}$ are much larger than 1.

\section{Mismatched ML Receiver}

We consider a mismatched ML receiver that uses the estimated channel instead of the true channel. In this way, the ML detection rule of the matrix $\mathbf{B}$ in (5a) is given by

$$
\hat{\mathbf{B}}=\arg \min _{\widetilde{\mathbf{B}}}\left\|\mathbf{r}-\sqrt{E_{\mathrm{s}}} \widetilde{\mathbf{B}} \hat{\mathbf{h}}\right\|^{2} .
$$

Taking (2) into account, it is readily verified that the detection algorithm (13) reduces to symbol-by-symbol detection for the different information symbols $s_{i}$ comprised in the matrix B:

$$
\hat{s}_{i}=\arg \min _{\widetilde{s}}\left|u_{i}-\tilde{s}\right|, \quad 1 \leq i \leq N_{\mathrm{s}},
$$


where the minimization is over all symbols $\tilde{s}$ belonging to the considered symbol constellation and the decision variable $u_{i}=u_{i, \mathrm{R}}+j u_{i, \mathrm{I}}$, with $u_{i, \mathrm{R}}$ and $u_{i, \mathrm{I}}$ denoting the real and imaginary parts of $u_{i}$, respectively, is given by

$$
u_{i}=\frac{\hat{\mathbf{h}}^{H}\left(\mathbf{C}_{i}^{*} \otimes \mathbf{I}_{L_{\mathrm{r}}}\right) \mathbf{r}+\mathbf{r}^{H}\left(\mathbf{C}_{i}^{\prime T} \otimes \mathbf{I}_{L_{\mathrm{r}}}\right) \hat{\mathbf{h}}}{\lambda \sqrt{E_{\mathrm{s}}}\|\hat{\mathbf{h}}\|^{2}} .
$$

\section{BIT ERROR RATE ANALYSIS}

In this section, we derive a closed-form BER expression for square OSTBCs under correlated Rayleigh fading with LMMSE channel estimation, using the particular channel decomposition (8) and the high-SNR approximation (11) of the channel error covariance matrix (10). In section VI, however, Monte-Carlo simulations show that this BER expression can be used also to obtain very accurate BER results over a wide range of SNRs, for both LMMSE and LS channel estimation.

Using (8) and denoting the LMMSE channel estimate (7) by $\hat{\mathbf{h}}$ for notational convenience, the received signal (5a) can be written as

$$
\mathbf{r}=\sqrt{E_{\mathrm{s}}} \mathbf{B} \hat{\mathbf{h}}+\sqrt{E_{\mathrm{s}}} \mathbf{B} \boldsymbol{\varepsilon}+\mathbf{w},
$$

where $\sqrt{E_{\mathrm{s}}} \mathbf{B} \hat{\mathbf{h}}$ is the useful component, $\mathbf{w}$ is the Gaussian channel noise, and $\sqrt{E_{\mathrm{s}}} \mathbf{B} \varepsilon$ is additional noise caused by the channel estimation error. It can be shown that the additional noise vector $\sqrt{E_{\mathrm{s}}} \mathbf{B} \varepsilon$ is Gaussian when conditioned on the data symbol vector $\mathbf{s}$. Using (16), the decision variable (15) can be expanded as

$$
u_{i}=s_{i}+n_{i}, \quad 1 \leq i \leq N_{\mathrm{s}},
$$

where the disturbance term $n_{i}$ contains contributions from the channel noise $\mathrm{w}$ and the channel estimation error $\varepsilon$, and is Gaussian when conditioned on s and $\hat{\mathbf{h}}$. In case of PCSI, the channel estimation error $\varepsilon=\mathbf{0}$ and $n_{i}$ is a ZM CSCG RV independent of $\mathbf{s}$ with variance $N_{0} /\left(\lambda E_{\mathrm{s}}\|\mathbf{h}\|^{2}\right)$.

Let us focus our attention to square $M$-QAM transmission with Gray mapping, which reduces to $\sqrt{M}$-PAM transmission for both the in-phase and quadrature information bits. Due to this fact, the BER for $M$-PAM with Gray mapping can be obtained in a very similar way. It can be shown that for square $M$-QAM and equally likely symbol vectors $\mathbf{s}$, the BERs related to the in-phase and quadrature bits of $s_{i}, 1 \leq i \leq N_{\mathrm{s}}$, are equal and independent of the index $i$. Hence, the average BER of a square OSTBC can be calculated as the BER related to the in-phase information bits of a data symbol $s_{i}$ in the transmitted code matrix $\mathbf{C}$, regardless of $i$. A decision error will occur when the real part $u_{i, \mathrm{R}}$ of the decision variable (15) is located inside the decision area of a QAM symbol $b \neq s_{i}$. Denoting by $b_{\mathrm{R}}$ and $b_{\mathrm{I}}$ the real and imaginary parts of the QAM symbol $b$, the projection on the real axis of the decision area of the symbol $b$ is referred to as the decision region of $b_{\mathrm{R}}$. We can write the BER as

$$
\begin{aligned}
\mathrm{BER}=\frac{1}{M^{N_{\mathrm{s}}}} \sum_{\mathbf{s} \in \Psi^{N_{\mathrm{s}}}} \sum_{b_{\mathrm{R}} \in \Psi_{\mathrm{R}}} \frac{d_{\mathrm{H}}\left(s_{i, \mathrm{R}}, b_{\mathrm{R}}\right)}{\frac{1}{2} \log _{2} M} \\
\mathbb{E}_{\hat{\mathbf{h}}}\left[P_{i, \mathrm{R}}\left(\mathbf{s}, b_{\mathrm{R}}, \hat{\mathbf{h}}\right)\right],
\end{aligned}
$$

where $\Psi$ and $\Psi_{\mathrm{R}}$ are the sets of the $M$-QAM constellation points and their real parts, respectively, $d_{\mathrm{H}}\left(s_{i, \mathrm{R}}, b_{\mathrm{R}}\right)$ is the Hamming distance between the in-phase bits allocated to the transmitted symbol $s_{i}$ and the detected symbol $b$, and $P_{i, \mathrm{R}}\left(\mathbf{s}, b_{\mathrm{R}}, \hat{\mathbf{h}}\right)$ is the probability that $u_{i, \mathrm{R}}$ is located inside the decision area of $b_{\mathrm{R}}$, when $\mathbf{s}$ and $\hat{\mathbf{h}}$ are known. When $d_{1}\left(s_{i, \mathrm{R}}, b_{\mathrm{R}}\right)$ and $d_{2}\left(s_{i, \mathrm{R}}, b_{\mathrm{R}}\right)$ denote the distances between $s_{i, \mathrm{R}}$ and the boundaries of the decision area of $b_{\mathrm{R}}$, with $d_{1}\left(s_{i, \mathrm{R}}, b_{\mathrm{R}}\right)<d_{2}\left(s_{i, \mathrm{R}}, b_{\mathrm{R}}\right), P_{i, \mathrm{R}}\left(\mathbf{s}, b_{\mathrm{R}}, \hat{\mathbf{h}}\right)$ reduces to

$$
P_{i, \mathrm{R}}\left(\mathbf{s}, b_{\mathrm{R}}, \hat{\mathbf{h}}\right)=Q\left(\frac{d_{1}\left(s_{i, \mathrm{R}}, b_{\mathrm{R}}\right)}{\sigma_{i, \mathrm{R}}(\mathbf{s}, \hat{\mathbf{h}})}\right)-Q\left(\frac{d_{2}\left(s_{i, \mathrm{R}}, b_{\mathrm{R}}\right)}{\sigma_{i, \mathrm{R}}(\mathbf{s}, \hat{\mathbf{h}})}\right),
$$

where $\sigma_{i, \mathrm{R}}(\mathbf{s}, \hat{\mathbf{h}})$ denotes the standard deviation of the real part of $n_{i}$ and $Q($.$) is the Gaussian Q$-function [10, Eq. (4.1)]. Note that we set $d_{2}\left(s_{i, \mathrm{R}}, b_{\mathrm{R}}\right)=\infty$, when $b$ is an outer constellation point. Assuming that the high-SNR approximation (11) is valid, it can be shown that the variance $\sigma_{i, \mathrm{R}}^{2}(\mathbf{s}, \hat{\mathbf{h}})$ of the real part of $n_{i}$ is given by

$$
\sigma_{i, \mathrm{R}}^{2}(\mathbf{s}, \hat{\mathbf{h}})=\frac{N_{0}}{2 \lambda E_{\mathrm{s}}\|\hat{\mathbf{h}}\|^{2}}\left(1+\frac{\lambda\|\mathbf{s}\|^{2}}{\gamma K_{\mathrm{p}}}\right) .
$$

Since $\sigma_{i, \mathrm{R}}^{2}(\mathbf{s}, \hat{\mathbf{h}})$ depends on the channel estimate $\hat{\mathbf{h}}$ through its squared norm $\|\hat{\mathbf{h}}\|^{2}$ only, a closed-form expression for (18) can be derived by averaging the $Q$-functions in (19) over the statistics of $\|\hat{\mathbf{h}}\|^{2}$. Denoting by $\lambda_{m}, m=1,2, \ldots, \kappa$, the $m$-th distinct eigenvalue of $\boldsymbol{\mathcal { R }}_{\hat{\mathrm{h}} \hat{\mathrm{h}}}$ given by (9), with corresponding algebraic multiplicity $c_{m}$, the probability density function (PDF) of $\|\hat{\mathbf{h}}\|^{2}$ is given by [11]

$$
p_{\|\hat{\mathbf{h}}\|^{2}}(x)=\sum_{m=1}^{\kappa} \sum_{n=1}^{c_{m}} D_{m, n} \frac{x^{n-1} \exp \left(-\frac{x}{\lambda_{m}}\right)}{(n-1) !\left(\lambda_{m}\right)^{n}},
$$

where $x \geq 0$. In (21), the parameters $D_{m, n}$ are given by

$$
D_{m, n}=\left.\frac{\left(\lambda_{m}\right)^{n-c_{m}}}{\left(c_{m}-n\right) !}\left[\frac{\mathrm{d}^{c_{m}-n}}{\mathrm{~d} s^{c_{m}-n}} \Psi_{m}(s)\right]\right|_{s=-\frac{1}{\lambda_{m}}},
$$

where

$$
\Psi_{m}(s)=\prod_{\substack{l=1 \\ l \neq m}}^{\kappa}\left(1+\lambda_{l} s\right)^{-c_{l}} .
$$

Using the PDF (21) and the result from [12, 14.4-15], it is easily shown that averaging the $Q$-functions in (19) over the statistics of $\|\hat{\mathbf{h}}\|^{2}$ yields

$$
\begin{aligned}
\mathbb{E}_{\hat{\mathbf{h}}}\left[Q\left(\frac{d_{q}\left(s_{i, \mathrm{R}}, b_{\mathrm{R}}\right)}{\sigma_{i, \mathrm{R}}(\mathbf{s}, \hat{\mathbf{h}})}\right)\right]=\sum_{m=1}^{\kappa} \sum_{n=1}^{c_{m}} D_{m, n} \\
\times\left[\frac{1-\mu_{m}}{2}\right]^{n} \sum_{k=0}^{n-1}\left(\begin{array}{c}
n-1+k \\
k
\end{array}\right)\left[\frac{1+\mu_{m}}{2}\right]^{k},
\end{aligned}
$$

where $q=\{1,2\}$, and $\mu_{m}$ is given by

$$
\mu_{m} \triangleq\left[\frac{\lambda d_{q}^{2}\left(s_{i, \mathrm{R}}, b_{\mathrm{R}}\right) \frac{E_{\mathrm{s}}}{N_{0}}\left(1+\frac{\lambda\|\mathbf{s}\|^{2}}{\gamma K_{\mathrm{p}}}\right)^{-1} \lambda_{m}}{1+\lambda d_{q}^{2}\left(s_{i, \mathrm{R}}, b_{\mathrm{R}}\right) \frac{E_{\mathrm{s}}}{N_{0}}\left(1+\frac{\lambda\|\mathbf{s}\|^{2}}{\gamma K_{\mathrm{p}}}\right)^{-1} \lambda_{m}}\right]^{\frac{1}{2}} .
$$


From (19) and (24), the closed-form BER expression for (18) is easily obtained.

\section{NumERICAL RESULTS}

In this section, we present numerical results from evaluating the presented closed-form BER expression under the assumption that $E_{\mathrm{p}}=E_{\mathrm{s}}$. Additionally, Monte-Carlo simulations indicate that the BER expression is not only asymptotically exact but also yields very accurate BER results for low to moderate SNR, for both LMMSE and LS channel estimation.

Fig. 1 displays the BER for the $4 \times 4$ OSTBC given by [5]

$$
\mathcal{C}_{4 \times 4}=\frac{2}{\sqrt{3}}\left(\begin{array}{cccc}
s_{1} & -s_{2}^{*} & -s_{3}^{*} & 0 \\
s_{2} & s_{1}^{*} & 0 & -s_{3}^{*} \\
s_{3} & 0 & s_{1}^{*} & s_{2}^{*} \\
0 & s_{3} & -s_{2} & s_{1}
\end{array}\right),
$$

where the scaling factor $\frac{2}{\sqrt{3}}$ is applied in order that (26) satisfies (2). The BER curves are shown for 4-QAM and 64-QAM constellations under correlated and uncorrelated Rayleigh fading, for $K=200$ and $K_{\mathrm{p}}=16$. Also shown are the BER results for PCSI, with $K=200$ and $K_{\mathrm{p}}=0$. We consider a single-antenna receiver and a covariance matrix $\mathcal{R}_{\mathrm{hh}}$, which in case of correlated fading is given by

$$
\boldsymbol{R}_{\mathbf{h h}}=\left(\begin{array}{cccc}
1 & 0.7 & 0.5 & 0.3 \\
0.7 & 1 & 0.7 & 0.5 \\
0.5 & 0.7 & 1 & 0.7 \\
0.3 & 0.5 & 0.7 & 1
\end{array}\right)
$$

Note that $\boldsymbol{\mathcal { R }}_{\mathbf{h h}}$ has a Toeplitz structure, which corresponds to the practical situation of an equally spaced linear antenna array [13]. Monte-Carlo simulations for the mismatched receiver with LMMSE channel estimation confirm the accuracy of the presented BER expression. From the figure, we observe that antenna correlation and ICE both give rise to a horizontal shift of the BER curve at high SNR, and that the amount of degradation due to ICE is more or less independent of the antenna correlation and the constellation size.

Fig. 2 shows the BER results for the $2 \times 2$ Alamouti code, which is given by [3]

$$
\mathcal{C}_{2 \times 2}=\left(\begin{array}{cc}
s_{1} & -s_{2}^{*} \\
s_{2} & s_{1}^{*}
\end{array}\right) .
$$

Assuming a dual-antenna receiver $\left(L_{\mathrm{r}}=2\right)$, Fig. 2 shows the BER curves for square $M$-QAM transmission, with $M \in\{4,16,64,256\}$, under correlated Rayleigh fading, for $K=100$ and $K_{\mathrm{p}}=14$. Also shown are the BER results for PCSI, with $K=100$ and $K_{\mathrm{p}}=0$. For correlated fading, the covariance matrix $\mathcal{R}_{\mathbf{h h}}$ is assumed to be given by $\boldsymbol{\mathcal { R }}_{\mathrm{hh}}=\mathcal{R}_{\mathrm{t}} \otimes \mathcal{R}_{\mathrm{r}}$, where $\boldsymbol{\mathcal { R }}_{\mathrm{t}}$ and $\boldsymbol{\mathcal { R }}_{\mathrm{r}}$ are given by

$$
\begin{aligned}
& \boldsymbol{R}_{\mathrm{t}}=\left(\begin{array}{cc}
1 & 0.3+j 0.1 \\
0.3-j 0.1 & 1
\end{array}\right), \\
& \boldsymbol{\mathcal { R }}_{\mathrm{r}}=\left(\begin{array}{cc}
1 & 0.4-j 0.6 \\
0.4+j 0.6 & 1
\end{array}\right) .
\end{aligned}
$$

Monte-Carlo simulations conducted for a mismatched receiver performing either LMMSE or LS channel estimation, indicate

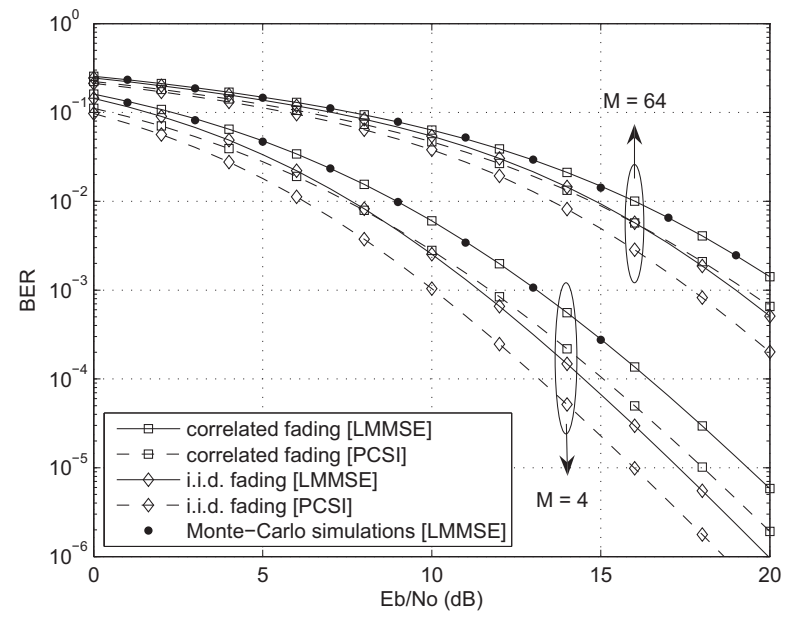

Fig. 1. BER of OSTBC given by (26) with $M$-QAM transmission, for correlated and i.i.d. Rayleigh fading, and for LMMSE channel estimation and PCSI.

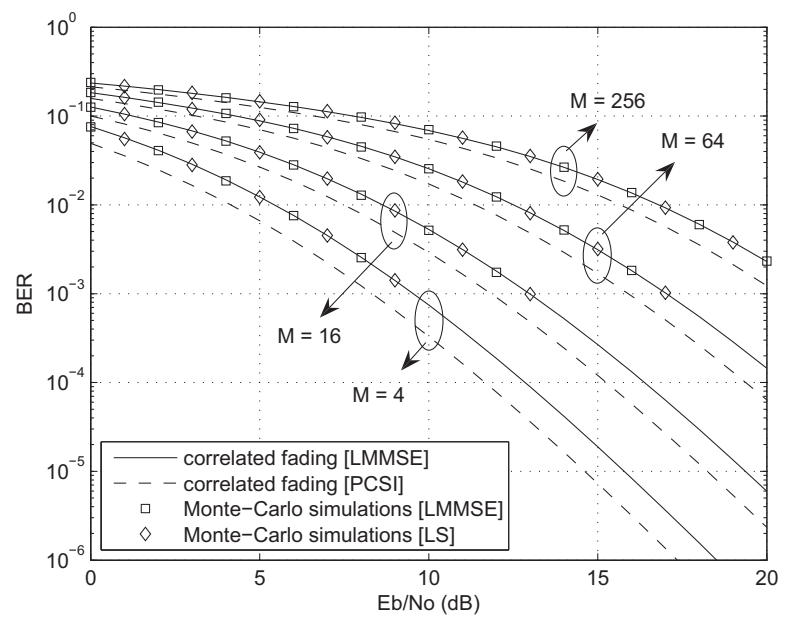

Fig. 2. BER of Alamouti's code with $M$-QAM transmission, for correlated Rayleigh fading, and for LMMSE channel estimation and PCSI. Monte-Carlo simulations indicate the accuracy of the presented BER expression for both LMMSE and LS channel estimation

that the presented BER expression yields very accurate BER results for LMMSE and LS channel estimation, in the range from low to high SNR.

\section{CONCLUSIONS}

In this work, we examined the BER performance of square orthogonal space-time block codes under arbitrarily correlated Rayleigh fading channels with LMMSE channel estimation. We presented a closed-form BER expression which is asymptotically exact and yields very accurate BER results also in the low to moderate SNR region, for both LMMSE and LS channel estimation. 


\section{ACKNOWLEDGMENTS}

The authors wish to acknowledge the activity of the Network of Excellence in Wireless COMmunications NEW$\mathrm{COM}++$ of the European Commission (contract n. 216715) that motivated this work. The first author also gratefully acknowledges the support from the Fund for Scientific Research in Flanders (FWO-Vlaanderen).

\section{REFERENCES}

[1] G. Foschini, "Layered space-time architecture for wireless communication in a fading environment when using multi-element antennas," Bell Labs Technical Journal, vol. 1, no. 2, pp. 41-59, Autumn 1996.

[2] E. Telatar, "Capacity of multi-antenna gaussian channels," European Transactions on Telecommunications, vol. 10, pp. 585-595, 1999.

[3] S. M. Alamouti, "A simple transmit diversity technique for wireless communications," IEEE J. Select. Areas Commun., vol. 16, pp. 14591478, Oct. 1998.

[4] V. Tarokh, H. Jafarkhani, and A. R. Calderbank, "Space-time block codes from orthogonal designs," IEEE Trans. Inform. Theory, vol. 45, no. 5, pp. 1456-1467, Jul. 1999.

[5] X.-B. Liang, "Orthogonal designs with maximal rates," IEEE Trans. Inform. Theory, vol. 49, no. 10, pp. 2468-2503, Oct. 2003.

[6] I.-M. Kim, "Exact BER analysis of OSTBCs in spatially correlated MIMO channels," IEEE Trans. Commun., vol. 54, no. 8, pp. 1365-1373, Aug. 2006.

[7] Y. Ma and L. Zhao, "Achievable performance of orthogonal STBC over spatially correlated rician channels," IEEE Trans. Veh. Technol., vol. 56, no. 3, pp. $1251-1261$, may 2007.

[8] L. Jacobs and M. Moeneclaey, "Effect of MMSE channel estimation on BER performance of orthogonal space-time block codes in Rayleigh fading channels," IEEE Trans. Commun., vol. 57, no. 5, pp. 1242-1245, May 2009.

[9] G. Taricco, "Optimum receiver design and performance analysis of arbitrarily correlated rician fading MIMO channels with imperfect channel state information," IEEE Trans. Inform. Theory, vol. 56, no. 3, pp. 1114-1134, Mar. 2010.

[10] M. K. Simon and M.-S. Alouini, Digital Communication over Fading Channels, 2nd ed. New York: Wiley, 2005.

[11] C. Siriteanu and S. Blostein, "Performance and complexity analysis of eigencombining, statistical beamforming, and maximal-ratio combining," IEEE Trans. Veh. Technol., vol. 58, no. 7, pp. 3383-3395, Sep. 2009.

[12] J. Proakis, Digital Communications, 4th ed. McGraw-Hill, 2001.

[13] G. C. Alexandropoulos, N. C. Sagias, F. I. Lazarakis, and K. Berberidis, "New results for the multivariate Nakagami- $m$ fading model with arbitrary correlation matrix and applications," IEEE Trans. Wireless Commun., vol. 8, no. 1, pp. 245-255, Jan. 2009. 